\title{
Tongue Movement and Role of Frenulum Linguae Effecting Tongue Movement during Prey Capture in Rana nigromaculata
}

\author{
*Correspondence to: \\ Lee $\mathrm{JH}$, \\ Tel: +82-55-249-2243 \\ Fax: +82-505-999-2150 \\ E-mail: jhlee@kyungnam.ac.kr
}

Received April 14, 2015

Revised June 22, 2015

Accepted June 23, 2015

\author{
So-Yeon $\mathrm{Im}^{1,3}$, Soo-Hyoun Je $\mathrm{J}^{2,3}$, Jung-Hun Lee ${ }^{4, *}$ \\ ${ }^{1}$ Masan Eui-shin Girls' Middle School, Changwon 631-851, Korea \\ ${ }^{2}$ Gyeryong Middle School, Geoje 656-814, Korea \\ ${ }^{3}$ Institute of Gifted Education in Science, Kyungnam University, Changwon 631-701, Korea \\ ${ }^{4}$ Department of Science Education, Kyungnam University, Changwon 631-701, Korea
}

\begin{abstract}
This study investigated the tongue movement during prey capture by Rana nigromaculata and the location of the frenulum linguae, which effects tongue movement. The tongue of $R$. nigromaculata are elliptical at the anterior and concave $U$-shaped at the posterior. The location of the frenulum linguae of $R$. nigromaculata is located at the front of the submentalis. This is due to the location of the frenulum linguae, which has significantly effects the movement and the expandability of the tongue. In terms of mobility, the tongue of $R$. nigromaculata and the expandability of the frenulum linguae allow the tongue to increase in length two or more times compared to immobile tongue in the other species. The time required until the swallowing movement after looking at and capturing the prey was 0.692000 seconds. Filming prey capture with a high speed camera showed that the rear part of the U-shaped tongue captures the prey with an appearance of stretching out longer to the front of tongue and turning in a reverse direction to bring the prey to the mouth.
\end{abstract}

Key Words: Rana nigromaculata, Tongue movement, Frenulum linguae

\section{INTRODUCTION}

Amphibians are remarkable in having a tongue that is propelled from the mouth, to impact on, adhere to, and pull prey into the buccal cavity (Regal \& Gans, 1976). While tongue shape and lingual papillae of vertebrata show many differences between species, the tongue is closely related to the habitat and food types (Lee \& Hahm, 2000). Besides, the tongue, which plays a critical role in food intake by vertebrates, exhibits significant morphological variations that appear to represent adaptation to the current environmental conditions of each respective habitat (Iwasaki, 2002).

The mechanical basis of tongue projection has long been discussed and debated. Regal (1966) and Özeti and Wake (1969) reported the mechanism of the tongue of frogs, which is propelled by the rotation of "soft" tissues about the mandibular symphysis, which is hurled anteriorly by a forward shift of the hyobranchial skeleton. Various studies of prey capture behavior by frogs (Regal \& Gans, 1976; Emerson, 1977; Gans \& Gorniak, 1982a, 1982b; Nishikawa \& Roth, 1991; Deban \& Nishikawa, 1992) have led to the formulation of several models of tongue protraction, but scientific and experimental confirmation is still lacking. The feeding mechanism is clearly an important factor that determines the success of adaption of vertebrates to their environment and their persistence through procreation (Roth \& Wake, 1989). The scientific information related to the movement of tongue during prey capture of amphibia is mainly distinguished into 1) prey captures by simply stretching out of the tongue while capturing the prey (e.g., Rana nigromaculata), 2) suddenly swallowing the prey by opening the mouth (e.g., Bombina orientalis), and 3) ingesting the prey to the mouth using the fingers of the foreleg (e.g., Xenopus laevis).

Various studies of prey capture behavior in frogs (Gans, 1961, 1962; Regal \& Gans, 1976; Emerson, 1977; Gans \& Gorniak, 1982a, 1982b; Nishikawa \& Roth, 1991; Deban \&

(a) This is an open-access article distributed under the terms of the Creative Commons Attribution Non-Commercial License (http://creativecommons.org/licenses/by-nc/4.0) which permits unrestricted noncommercial use, distribution, and reproduction in any medium, provided the original work is properly cited.

Copyrights @ 2015 by Korean Society of Microscopy 
Nishikawa, 1992, Nishikawa et el., 1999) propose a pattern of tongue propulsion, but there are no reported studies on the specific mechanisms related to tongue movement and frenulum linguae of $R$. nigromaculata related to the prey capture. Accordingly, this study intends to find out about the movement of the tongue during prey capture by $R$. nigromaculata along with the location and the role of frenulum linguae having effect on the tongue movement.

\section{MATERIALS AND METHODS}

Ten individual male frogs of three species, $R$. nigromaculata, Rana catesbeiana, and B. orientalis, were gathered in April and May 2014.

The tongue movement of $R$. nigromaculata, was analyzed based on the morphological characteristics and the motion of tongue during prey capture. In this study, we hypothesized that the location of a band shaped muscle structure connecting the bottom of tongue and the mucous membrane of mouth called frenulum linguae would affect the movement of the tongue.

(1) To find out the tongue shape and the location of frenulum linguae, inhalation anesthesia was performed on each of the frogs ( $R$. nigromaculata, $R$. catesbeiana, and B. orientalis) using chloroform. Tweezers were inserted into the rear bottom section of the tongue, and the shape of the frenulum linguae was seen by pulling the tongue forward and removing.

(2) To find the movement of tongue during prey capture, actions were filmed at 2,000 fps (frames per second) using a high speed camera (Photron Fastcam SA 1.1; Photron, USA).

(3) To find the extensibility of tongue, the moving distance was measured by binding the front and the back of the removed tongue after weights were attached.
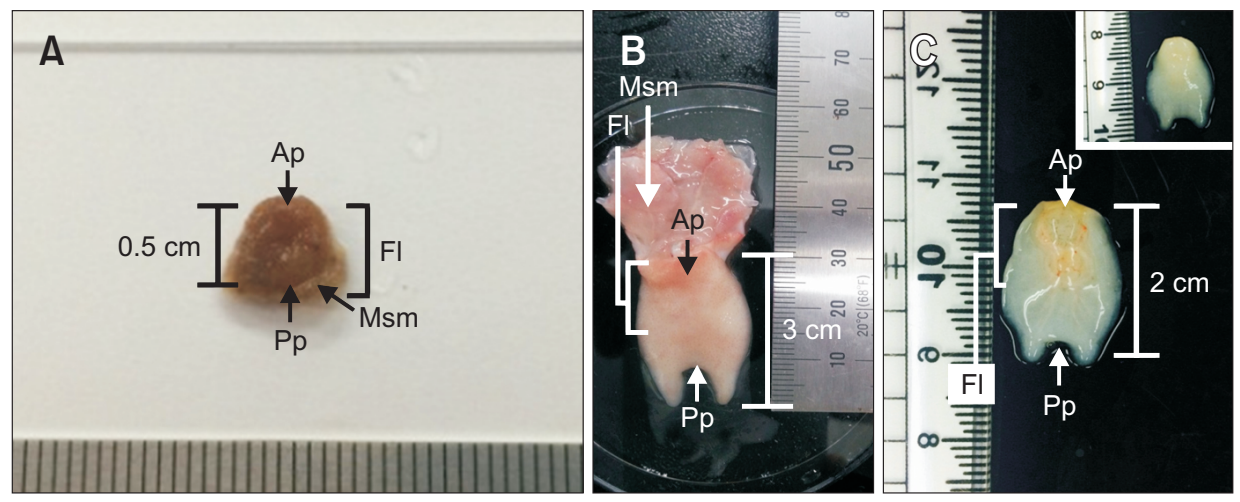

Fig. 1. External morphology of tongue and the location of frenulum linguae of Bombina orientalis (A, dorsal view), Rana catesbeiana (B, ventral view) and Rana nigromaculata (C, ventral view; inset, dorsal view). Ap, anterior part of tongue; $\mathrm{Fl}$, frenulum linguae; Msm, muscle submaxilla; Pp, posterior part of tongue.

A

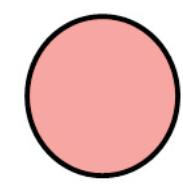

B

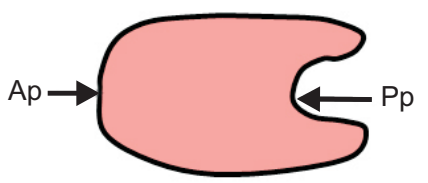

C

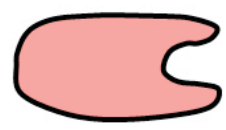

D

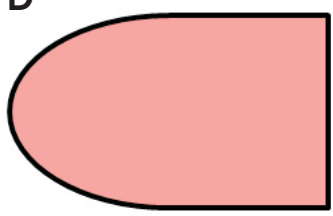

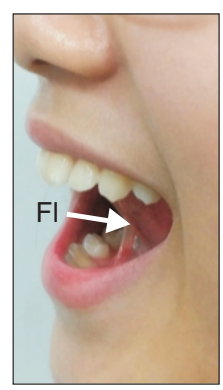

$\mathrm{H}$

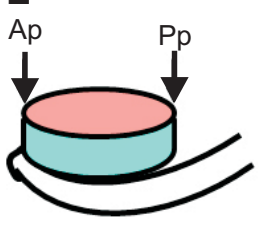

$\mathbf{F}$

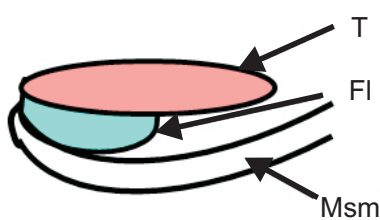

G

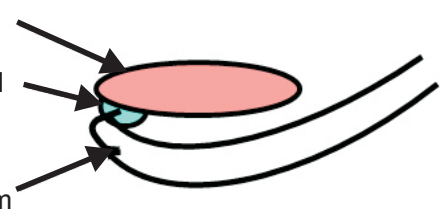

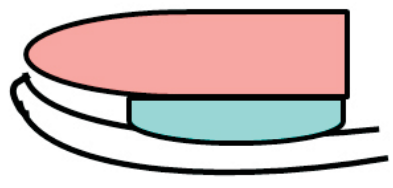

Fig. 2. Schematic representation of tongue shape and the location of frenulum linguae of Bombina orientalis (A, E), Rana catesbeiana (B, F), Rana nigromaculata (C, G), and human (D, H); dorsal view (A-D) and side view (E-H). Photograph showing the location of the frenulum linguae in a human. The location of the frenulum linguae, $B$. orientalis is extended to all areas that are in direct contact with the surface of submentalis muscle and the body of tongue. $R$. catesbeiana frenulum linguae is located near the middle of the body of tongue at the front part of the submentals. $R$. nigromaculata frenulum linguae located only at the front of submentalis. Human frenulum linguae is located near the rear two thirds at the back of submentalis. Ap, anterior part of tongue; Pp, posterior part of tongue; $\mathrm{Fl}$, frenulum linguae; T, tongue; Msm, muscle submaxilla. 


\section{RESULTS}

\section{Shape of Tongue and Location of Frenulum Linguae (Tongue Tie)}

While the tongue of B. orientalis is spherical (Fig. 1A, 2A), the tongue of $R$. catesbeiana (Fig. 1B, 2B) and R. nigromaculata
(Fig. 1C, 2C) are elliptical at the front, assuming a form of concave U-shape at the rear. The frenulum linguae, extended to all areas that are in direct contact with the surface of submentalis and the body of tongue in B. orientalis (Fig. 2E). The frenulum linguae was located near the middle of the body of tongue at the front of the submentalis in $R$. catesbeiana
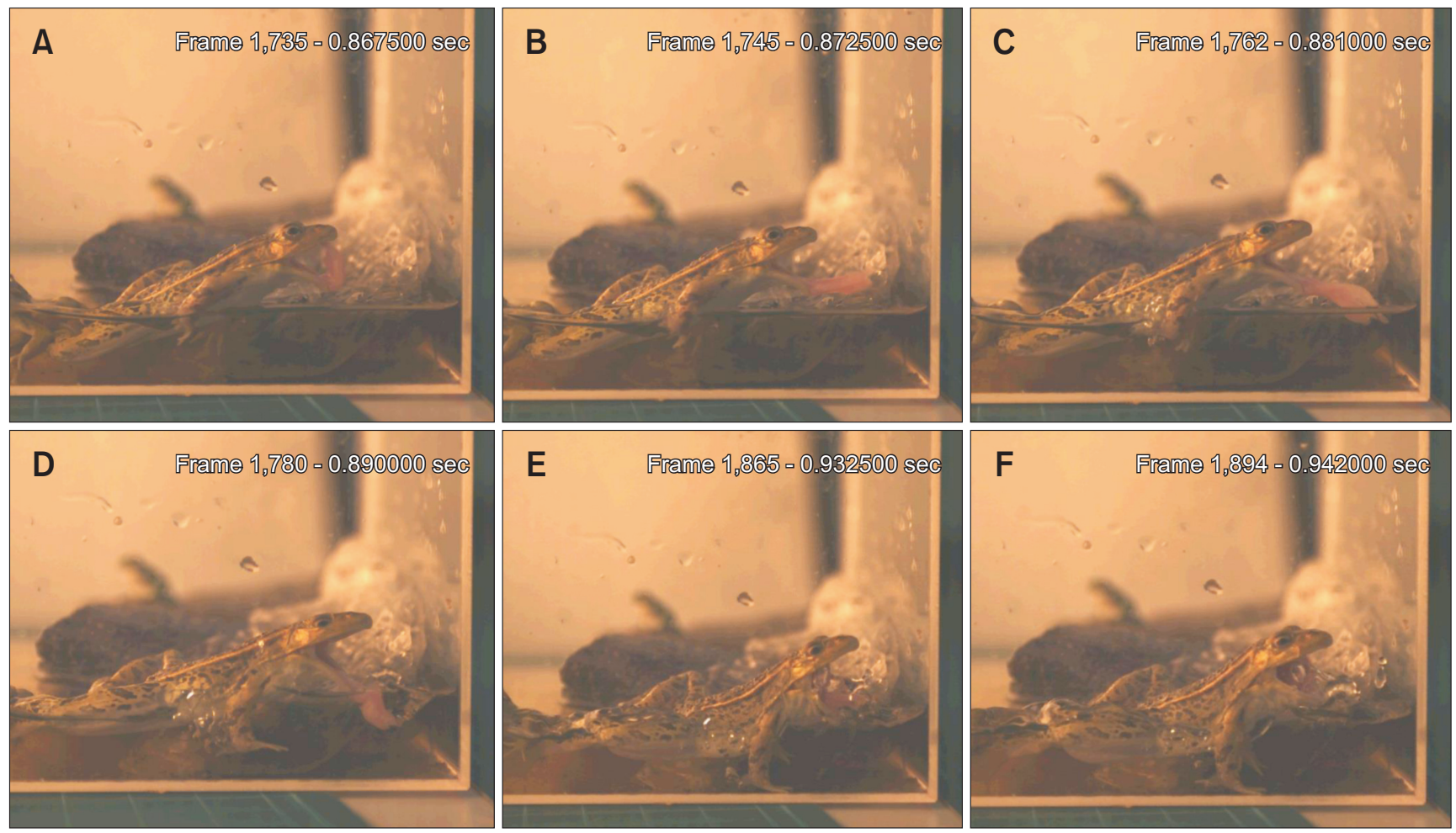

Fig. 3. High speed camera (2,000 fps) sequence of Rana nigromaculata eating an ant. Frame numbers on the upper right refer to time (sec) since the onset of mouth on the opening. Note that when the mouth opens fully the tongue rotates forward. fps, frames per second.
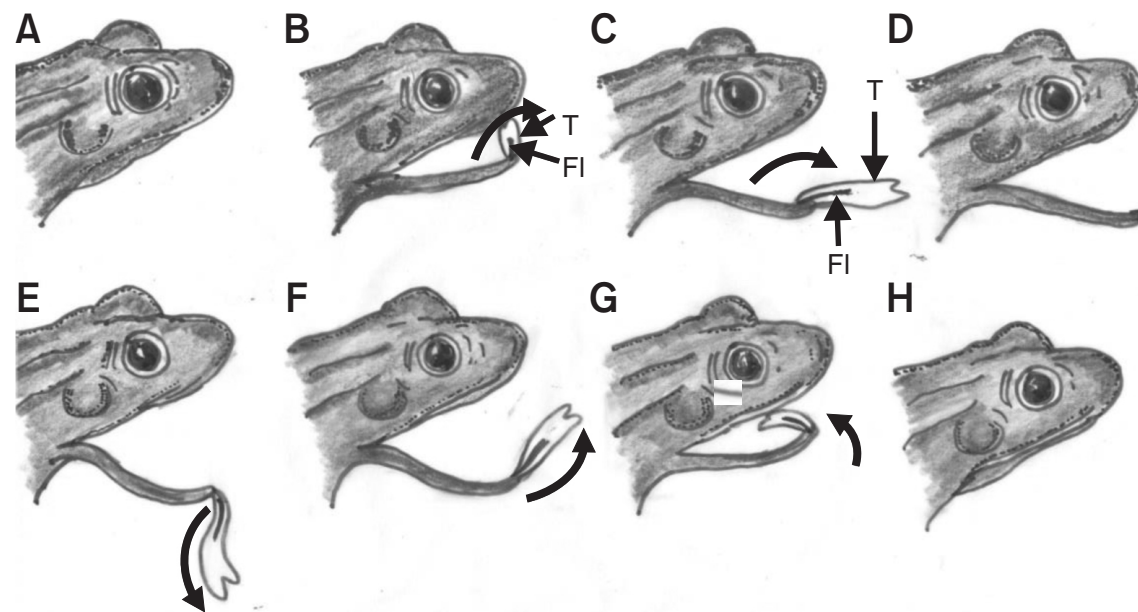

Fig. 4. Schematic representations of the tongue movement during prey capture of Rana nigromaculata. (A) Figure is prior to the prey capture. Fig. 4B to G shows the mouth opening fully and the tongue rotating forward. Fig. $4 \mathrm{H}$ is after prey capture. The arrow is the direction of tongue movement. The $\mathrm{U}$-shaped tongue captures the prey with an appearance of stretching out longer to the front of tongue and turning in a reverse direction while capturing the prey. (A) Frame 616, $0.308000 \mathrm{sec}$; (B) frame 1,735, $0.867500 \mathrm{sec}$; (C) frame 1,745, $0.872500 \mathrm{sec}$; (D) frame 1,762, $0.881000 \mathrm{sec}$; (E) frame 1,780, $0.890000 \mathrm{sec}$; (F) frame 1,865, $0.932500 \mathrm{sec}$; $(\mathrm{G})$ frame 1,894, $0.942000 \mathrm{sec}$; $\mathrm{H})$ frame 2,000, $1.000000 \mathrm{sec}$. T, tongue; Fl, frenulum linguae. 
(Fig. 2F) and located only at the front of the submentalis in $R$. nigromaculata (Fig. 2G). In the case of humans, the frenulum linguae was located about $2 / 3$ of the way to the rear of the tongue (Fig. 2H).

\section{Tongue Motility according to the Prey Capture}

To find out the mobility of the tongue when $R$. nigromaculata captures the prey, 2,000 fps filming, using a high speed camera (Photron Fastcam SA 1.1), indicated the process of tongue movement into each stage (Fig. 3, 4). Therefore, prey is integrated as the tongue stretches to the front (Fig. 3A-C, $4 \mathrm{~B}-\mathrm{D}$ ) and swallowing toward the mouth as the tongue rolls (Fig. 3D-F, 4E-G). The time (2,000 fps/frame 2,000, 1.000000 sec) required for swallowing (2,000 fps/frame 1,894, 0.942000 $\mathrm{sec})$ after looking at the prey (2,000 fps/frame 616, 0.308000 $\mathrm{sec})$, including capture (2,000 fps/frame 1,762, $0.881000 \mathrm{sec})$, was 0.692000 second.

\section{Extensibility of Tongue}

As shown in Fig. 5, weights (100, 200, 300, 400, 500 g) were attached to each removed tongue to find the extensibility of

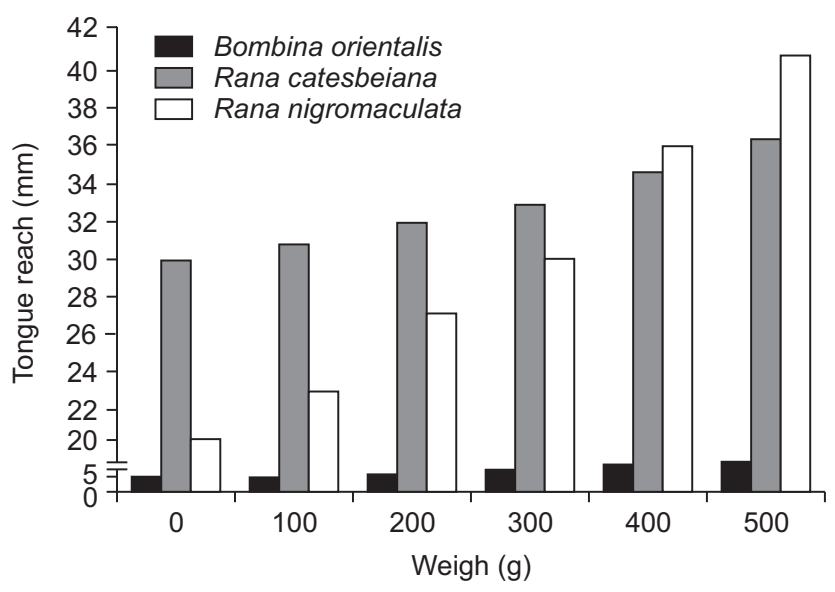

Fig. 5. Comparison of expansibility of the tongue of Bombina orientalis, Rana catesbeiana, and Rana nigromaculata by change weights. Note that the tongue expansibility of $R$. nigromaculata has increased about two times. the tongue, comparing the control group (B. orientalis and $R$. catesbeiana) and the experimental group ( $R$. nigromaculata). The tongue of $B$. orientalis did not extend much (increased to $10 \mathrm{~mm}$ with $500 \mathrm{~g}$ weight attached at the initial $5 \mathrm{~mm}$ ). $R$. catesbeiana increased from 30 to $36 \mathrm{~mm}$, while $R$. nigromaculata which is the species for this study, increased about two times (Fig. 5).

The tongue of $R$. nigromaculata extends twice or more in length compared to the immobile tongues of the controls (Figs. 5, 6).

\section{DISCUSSION}

Gans $(1961,1962)$ first proposed a model of tongue propulsion in a frog (Rana, an advanced frog). According to his proposal, tongue contraction turns the flaccid mass into a rigid rod or lever, and rapid depression of the attachment of the genioglossus with the mandibular symphysis rotates the short end of the lever over a rising fulcrum; this provides maximum linear acceleration of the distal genioglossal tip. Namely, the soft tissues of the lingual tip are carried along by the end of the genioglossal rod and expend their kinetic energy in further extension. Nevertheless, the models of the prey capture behavior in frogs ascribes roles in protraction to various muscles, including the $\mathrm{m}$. genioglossus medialis and basalis, the $\mathrm{m}$. submentalis, the $\mathrm{m}$. geniohyoideus medialis and lateralis, and the m. sternohyoideus (Regal \& Gans, 1976; Emerson, 1977; Gans \& Gorniak, 1982a, 1982b; Nishikawa \& Roth, 1991; Deban \& Nishikawa, 1992). On the other hand, the $\mathrm{m}$. genioglossus basalis and medialis and the $\mathrm{m}$. submentalis are all necessary for normal tongue protraction in the Gans and Gorniak (1982a, 1982b) model.

Nishikawa and Roth (1991) used high-speed videography to analyze the kinematics of prey capture before and after denervation and demonstrated that the $\mathrm{m}$. genioglossus is necessary for tongue protraction, while the $\mathrm{m}$. submentalis is necessary for mandibular bending, but not for tongue protraction.

In many anurans, the tongue shortens during protraction as $\mathrm{m}$. genioglossus contracts, pulling the tongue pad forwards
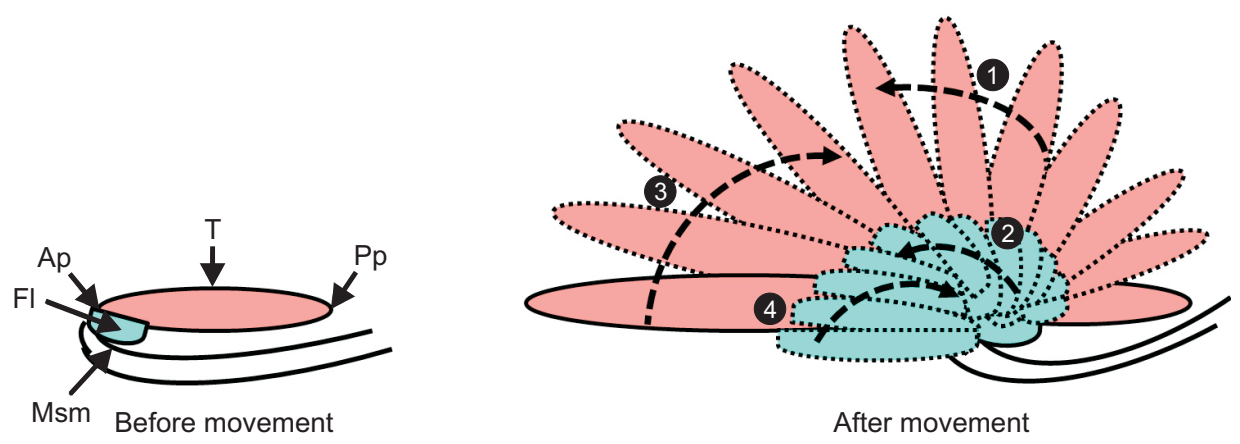

Fig. 6. Model of expandability of the tongue and the frenulum linguae. Note that the direction of motion of the tongue (1), (3) and frenulum linguae (2), (4)) during prey capture, respectively. The expandability of the frenulum linguae is increased by twice or more in length compared to an immobile. Ap, anterior part of tongue; $\mathrm{Fl}$, frenulum linguae; Msm, muscle submaxilla; Pp, posterior part of tongue; $\mathrm{T}$, tongue. 
towards the mandibular symphysis (Deban \& Nishikawa, 1992). Deban and Nishikawa (1992) reported that the m. genioglossus is necessary to produce complete tongue protraction and that the $\mathrm{m}$. submentalis is necessary for mandibular bending, but not necessary for complete tongue protraction in Hyla cinerea. According to Nishikawa and Gans (1996), tongue protraction occurs in two phases: an early phase during which the lingual tip moves upward and forward relative to the mandibular tip as the tongue shortens, and a later phase during which the lingual tip moves downward and forward relative to the mandibular tip, as the tongue elongates under its own momentum. Nishikawa et al. (1999) proposed the tongue protractor muscle consists of two parts, that is $\mathrm{m}$. genioglossus longitudinalis and $\mathrm{m}$. genioglossus dorsoventrlis. They suggested that the m. genioglossus dorsoventralis then plays the major role in tongue elongation. The goal of this study was to investigate morphological adaptations associated with tongue movement and the role of the frenulum linguae on the tongue movement during prey capture. While the tongue of $B$. orientalis is spherical, the tong-ue shapes of both $R$. catesbeiana and $R$. nigromaculata are elliptical in the front and concave U-shape at the rear (Lee et al., 1999; Lee \& Hahm, 2000), which is in agreement with previous published results.

The location of frenulum linguae, is extended to all areas that are in direct contact with the surface of submentalis muscle and the body of tongue in B. orientalis, is located near the middle of the body of tongue at the front part of submentalis in $R$. catesbeiana and located only at the front of submentalis in $R$. nigromaculata. Judging from these results, $B$. orientalis has a structure which is appropriate for opening up the mouth wide to swallow suddenly and is unable to stretch out the tongue. $R$. catesbeiana assumes a structure which is appropriate for suddenly swallowing the nearby prey, although it would stretch the tongue forward in case of capturing the prey located at longer capturing distance, as frenulum linguae was also located at the bottom of tongue. But in case of the tongue of $R$. nigromaculata, which is the species for this study, the frenulum linguae was touching the end of the front of the submentalis, so it could have a structure which allows longer tongue stretching.

A frog catches an insect by throwing its sticky tongue out of its mouth and wrapping it around the prey. The frog's tongue then snaps back and thrusts the food down its throat (http://www.kidzone.ws/lw/frogs/facts5.htm). The movement of the tongue occurs because the tongue is at the front of mandible without the rear end of the tongue being attached to the bottom of mouth; the tongue turns in a reverse direction as the front end is folded to the back side (Scienceall, Encyclopedia of Knowledge). According to the study, $R$. nigromaculata captures the prey with the rear part of the U-shaped tongue stretching out past the front of tongue and then turning in a reverse direction (Fig. 7). In other words, ingesting the prey occurs by stretching the back of the tongue toward the front and then swallowing occurs after the prey is brought to the pharynx by rolling the tongue (Fig. 7). This can occur because of the frenulum linguae location, the movement, and the expandability of the tongue (Fig. 6).

The action of the tongue when capturing prey depends on the location of the frenulum linguae. It was found that the tongue in $R$. nigromaculata was very flexible and expandable because of the frenulum linguae compared to the controls; the tongue stretched to over 2 times the length (Figs, 5, 6). Judging from the results, the location of frenulum linguae has a significant effect on tongue movement where capturing prey (Fig. 6).

The time required for capturing and swallowing the prey was 0.692000 second in $R$. nigromaculata (Fig. 4). The mobility and the directivity of the tongue of $R$. nigromaculata allows for the prey to be captured through a wide range of integration including the hypoglossal nerve (12th cranial nerve), which participates in swallowing, mastication, and mobility control of the tongue, the sensory nerve fiber from the lingual nerve, and the third neural network along with the facial nerve, sensing taste on the front $2 / 3$ of the tongue epithelium and the glossopharyngeal nerve dominating general perception and taste on the rear $1 / 3$ of the tongue epithelium all after identification of the location of prey using visual information

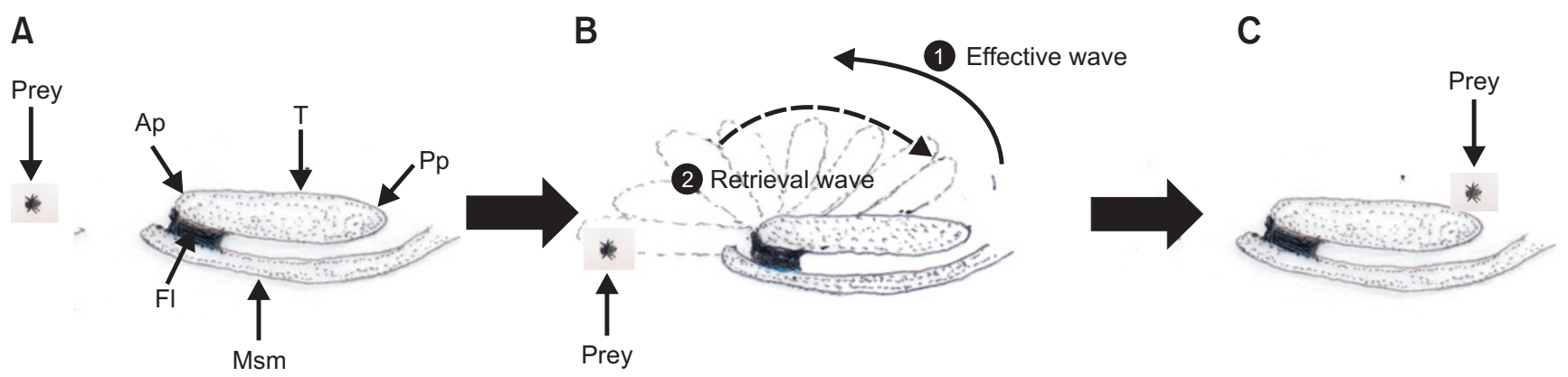

Fig. 7. Model of tongue movement and the direction of tongue movement during prey capture in Rana nigrpmaculata. Prey capture is performed by the stretching of the tongue toward the front and swallowing by rolling the tongue and bringing the prey to the pharynx. (A) Before movement. (B) During movement. (C) After movement. Ap, anterior part of tongue; Fl, frenulum linguae; Msm, muscle submaxilla; Pp, posterior part of tongue; T, tongue. 
(2nd cranial nerve).

\section{CONCLUSIONS}

This study has investigated the tongue mobility during prey capture by $R$. nigromaculata and the location of frenulum linguae (tongue tie), which effects tongue movement. While the tongue of $B$. orientalis is spherical, the tongue shapes of both $R$. catesbeiana and $R$. nigromaculata are elliptical at the anterior and concave U-shaped at the posterior. The location of the frenulum linguae, while it is extended to all areas that are in direct contact with the surface of submentalis muscle and the body of the tongue in the case of $B$. orientalis, is located near the middle of the body of the tongue at the front of the submentalis in R. catesbeiana, and is at the front of the submentalis in $R$. nigromaculata. This is due to the location of the frenulum linguae, which has significantly effects the movement and the expandability of the tongue. In terms of mobility, the tongue of $R$. nigromaculata and the expandability of the frenulum linguae allow the tongue to increase in length two or more times compared to immobile tongue in the other species. And the time (2,000 fps/frame 2,000, $1.000000 \mathrm{sec})$ required until the swallowing movement $(2,000 \mathrm{fps} /$ frame $1,894,0.942000 \mathrm{sec})$ after looking at $(2,000 \mathrm{fps} /$ frame 616 , $0.308000 \mathrm{sec})$ and capturing (2,000 fps/frame 1,762, 0.881000 sec) the prey was 0.692000 second. Filming prey capture with a high speed camera showed that the rear part of the U-shaped tongue captures the prey with an appearance of stretching out longer to the front of tongue and turning in a reverse direction to bring the prey to the mouth. Therefore, in case of the movement and the directivity of tongue according to the prey capture of $R$. nigromaculata, it is regarded that the prey is captured through a wide range of information including the hypoglossal nerve (12th cranial nerve) and the facial nerve participating in swallowing action, mastication and mobility control of the tongue or the glossopharyngeal nerve dominating general perception and taste sense, etc. after identifying the location of prey using visual information (2nd cranial nerve).

\section{CONFLICT OF INTEREST}

No potential conflict of interest relevant to this article was reported.

\section{REFERENCES}

Deban S M and Nishikawa K C (1992) The kinematics of prey capture and the mechanism of tongue protraction in the green tree frog, Hyla cinerea. J. Exp. Biol. 170, 235-256.

Emerson S B (1977) Movement of the hyoid in frogs during feeding. Am. J. Anat. 149, 115-120.

Gans C (1961) A bullfrog and its prey. A look at the bio-mechanics of jumping. Natural History. New York, 70:26-37.

Gans C (1962) The tongue protrusion mechanism in Rana catesbeana. (abst.). Amer. Zool. 2, 524.

Gans C and Gorniak G C (1982a) How does the toad flip its tongue? Test of two hypotheses. Science 216, 1335-1337.

Gans C and Gorniak G C (1982b) Functional morphology of lingual protrusion in marine toads (Bufo marinus). Am. J. Ant. 163, 195-222.

Iwasaki S (2002) Evolution of the structure and function of the vertebrate tongue. J. Anat. 201, 1-13.

Lee J H and Hahm K H (2000) A morphological study on the tongue of Rana catesbeiana. J. Basic. Sci. Res. Inst. 14, 155-170.

Lee J H, Son S W, and Heo J C (1999) Fine structure of the lingual papillae of Bombina orientalis and Rana catesbeiana. J. Innstit. Natur. Sci. 13, 99-108.

Nishikawa K C and Gans C (1996) Mechanisms of tongue protraction and narial closure in the marine toad Bufo marinus. J. Exp. Biol. 199,
2511-2529.

Nishikawa K C, Kier W M, and Smith K K (1999) Morphology and mechanism of tongue movement in the African pig-nosed frog Hemisus marmoratum: A muscular hydrostatic model. J. Exp. Biol. 202, 771-780.

Nishikawa K and Roth G (1991) The mechanism of tongue protraction during prey capture in the frog Discoglossus pictus. J. Exp. Biol. 159, 217-234.

Özeti N and Wake D B (1969) The morphology and evolution of the tongue and associated structures in salamanders and newts. (family Salamanderidae). Copeia 1969, 91-123.

Regal P (1966) Feeding specializations and the classification of terrestrial salamanders. Evolution 20, 392-407.

Regal P J and Gans C (1976) Functional aspects of the evolution of frog tongues. Evolution 30, 718-734.

Roth G and Wake D B (1989) Conservatism and innovation in the evolution of feeding in vertebrates. In: Complex Organismal Functions: Integration and Evolution in Vertebrates, eds. Wake D B and Roth G, pp. 7-21, (John Wiley \& Sons, New York).

Scienceall, Encyclopedia of Knowledge. "Tongue".

http://www.kidzone.ws/lw/frogs/facts5.htm "What's with that tongue?" 